\title{
An Investigation of Topologies and Migration Schemes for Asynchronous Distributed Evolutionary Algorithms
}

\author{
Muhannad Hijaze \\ School of MACS, Heriot-Watt University \\ Edinburgh, UK \\ mh132@hw.ac.uk
}

\author{
David Corne \\ School of MACS, Heriot-Watt University \\ Edinburgh, UK \\ dwcorne@macs.hw.ac.uk
}

\begin{abstract}
Distributed evolutionary algorithms are of increasing interest and importance for three main reasons: (i) a well designed dEA can outperform a 'standard' EA in terms of reliability, solution quality, and speed; (ii) they can (of course) be implemented on parallel hardware, and hence combine efficient utilization of parallel resources with very fast and reliable optimization; (iii) parallel hardware resources are increasingly common. A dEA operates as separate evolving populations with occasional interaction between them via 'migration'. A specific dEA is characterized by the topology and nature of these interactions. Although the field is sizeable, there is still relatively little exploration of the performance of alternative topologies and interaction mechanisms. In this paper we compare some simple, novel dEA topologies with the cube-based topology that forms the basis of Alba et al's GDRCGA (a state of the art dEA). We find the best results (when topologies are compared on a like for like basis in terms of number of processors) emerge from a three-level tree-based topology.
\end{abstract}

Keywords- function optimization, evolutionary algorithms, parallel evolutionary algorithms.

\section{INTRODUCTION}

Distributed evolutionary algorithms (dEAs) operate by having several independent populations of chromosomes, with occasional interaction between them. In a typical dEA, the separate populations will evolve independently for a number of generations, and then "migration" will occur, in which chromosomes from one or more of the populations (or 'demes') will be copied into one or more of the other populations. The populations then continue to evolve independently until the next migration event, and so on. Such an EA design is well known to have several desirable properties. Not least is the fact that several dEAs are well known to perform more successfully than standard EAs that are otherwise the same (e.g. in terms of operators and total population size) - that is, when implemented on a single processor, both solution quality and speed (in terms of total fitness evaluations) can be very favourable. Secondly, dEAs are of course highly parallelizable, and offer a highly natural way to exploit a variety of different parallel architectures. The latter advantage is becoming increasingly more important as parallel hardware resources

There are two main kinds of dEA (note that these are often simply called "parallel genetic algorithms). In the simplest, a standard EA is distributed over several processors but otherwise is little changed algorithmically. The alterations to the EA itself are only those necessary, if any, to enable exploitation of the parallel hardware. Examples include $[1,2]$.

The second (and main) line of research in distributed EAs involves the establishment of (largely) independent (sub)populations, each using its own processor. In such a scheme, a 'migration' strategy is used to communicate information between processors at intervals [3]. There are a great variety of alternative migration schemes. The migration strategy is typically to copy good chromosomes from some populations to others. The broad dynamics of such a dEA amount to healthy forms of exploration (promoted by independent subpopulations, mostly non-interacting) and exploitation (promoted by migration) that are not shared by single-population EAs. As mentioned, this typically leads to improved performance in terms of both solution quality and speed.

Such dEAs are often termed either fine-grained or coarse-grained, depending on the sizes of the subpopulations. The 'ultimate' fine-grained models have a single individual per processor (e.g. [4]). A more favoured approach, which is more in tune with the majority of hardware configurations, is the coarse grained model (e.g. [5-9]. The Genitor algorithm [8] is a particularly wellknown example, in which the subpopulations were linked in a ring topology - migration events involved chromsomes being copied from a population to its immediate neighbours in the ring. A more recent example is that of [7], in which the populations are linked in a cube topology, in which subpopulations are vertices of the cube, and each is linked to three others along the edges of the cube. We explore such a cube topology in this paper; the dGA model explored in [7] has several other sophistications; we do not implement those here, being interested for current purposes only in the performance of alternative simple topologies. 
Thread 1:

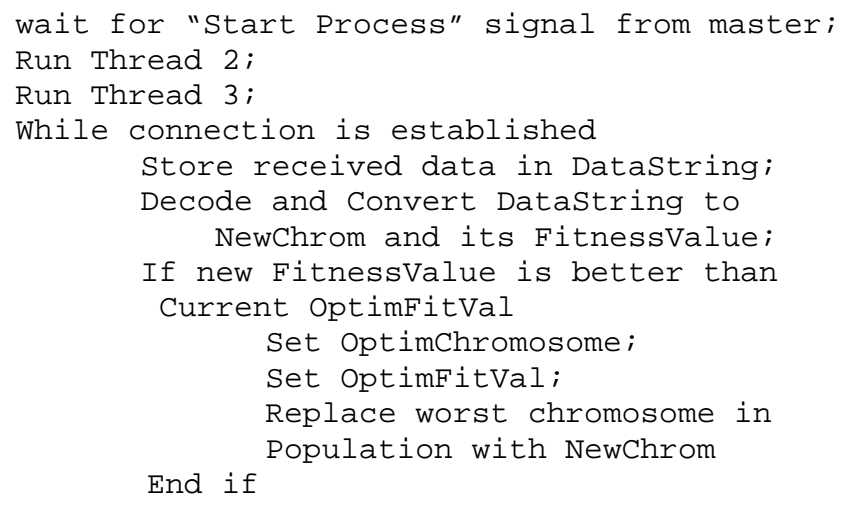

Thread 2:

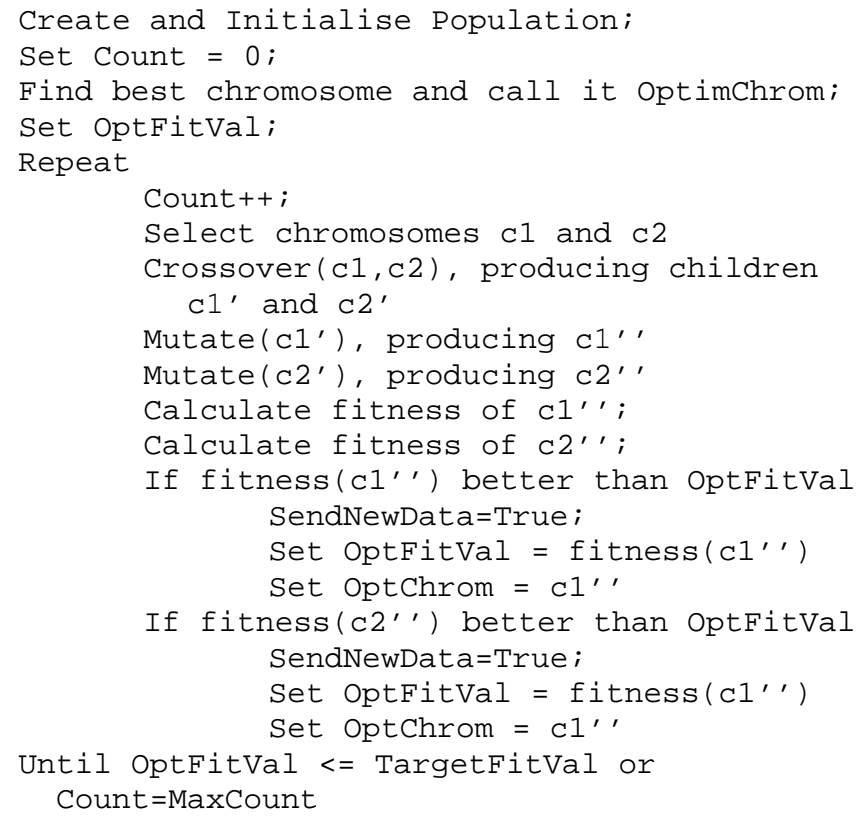

\section{B. Evolutionary Algorithm and Other Implementation Details}

In all models we use a generational evolutionary algorithm that operates with a truncation selection strategy [10], in which, in each generation, the best $33 \%$ of the population are retained, and, selecting only from this best 33\%, crossover and mutation are employed to generate the remaining $66 \%$ of the new population.

Our EA uses crossover and mutation operators detailed in [7]; specifically the fuzzy connective-based crossover operators: F- Crossover, S- Crossover, L- Crossover, MCrossover, along with one-point, two-point, and uniform crossover. Each time a crossover operator is applied, it is a uniform random choice between these seven. Mutation (Gaussian mutation of a single randomly chosen parameter) is performed on a child of crossover with probability 0.25 .

All our models use 15 individuals per subpopulation and (where applicable) a migration is performed every 25 generations. The probability of update an individual by mutation is 0.25 , and the crossover probability is 0.6 . There is a predefined maximum number of generations (10000), but a trial run will terminate if it has reached the target fitness values.

The physical hardware used is a cluster of eight personal computers running Microsoft widows XP Professional SP3, each one having an Intel Pentium IV 2.99 $\mathrm{GHz}$ processor and $2 \mathrm{~GB}$ of memory. The machines are interconnected by a Fast-Ethernet (100 Mbps) network.

\section{Topologies}

In model 1 (T1), the master connects directly to each of 16 clients, and clients operate two per processor (i.e. are distributed over 8 machines). Figure 1 illustrates model 1.

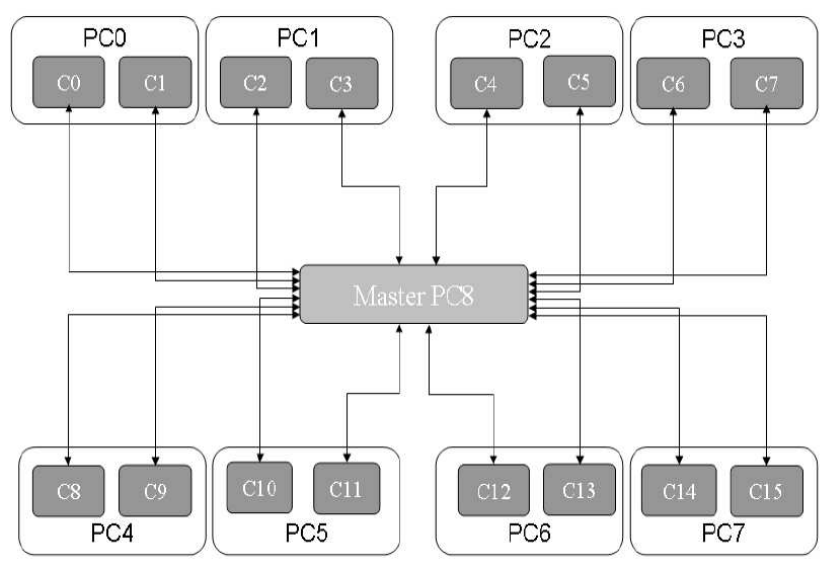

Figure 1. Model 1 topology for 16 clients. 


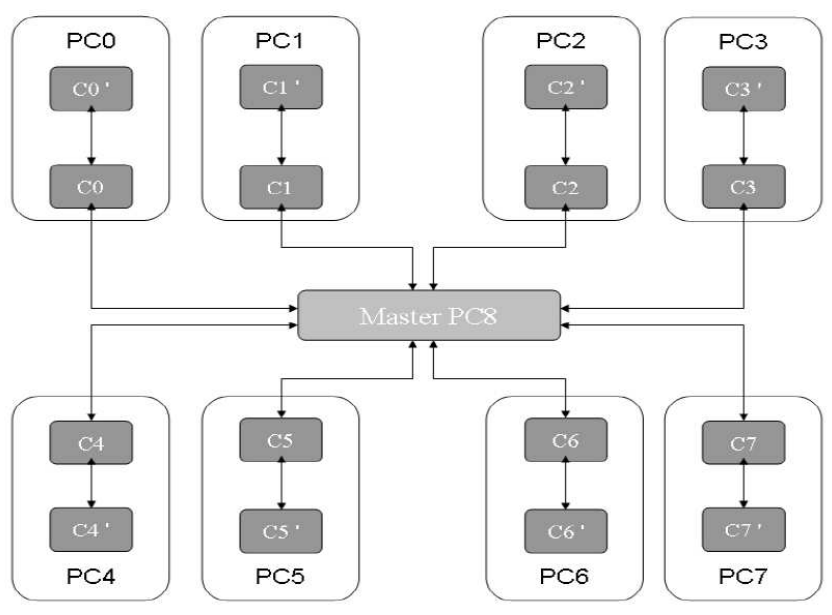

Figure 2. Model 2 topology for 16 clients.

Model 2 (T2) differs in a simple way from model 1, but in a way which leads to potentially quite different search dynamics. In Model 2, which is illustrated in Fig. 2., each master connects directly with each of eight groups, and each group consists of precisely two intercommunicating clients. Recall that, with the basic model (model 1), the master keeps track of the current overall optimum, and will copy this to the current worst client whenever a new optimum is discovered. This is so for model 2 at the level of groups; in model 2, a new best chromosome will first be transferred to the sister client in its group, and will soon appear in both clients of the previously worst group - hence there is, in some sense, more exploitation of newly discovered good chromosomes in model 2.

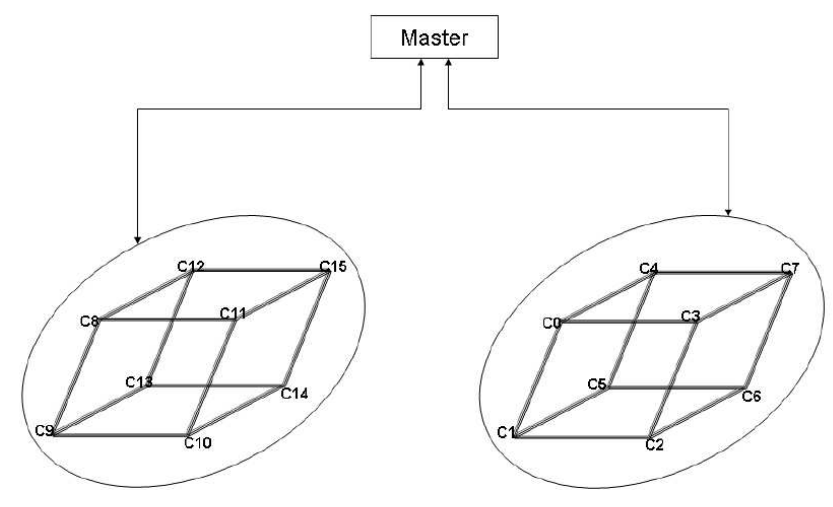

Figure 3. Model 3 topology for 16 clients.

In model 3 (T3), illustrated in Fig. 3., we base the topology and interactions on the model of Alba et al [7], in which a master controls two overall groups, but each group has a number of overlapping subgroups based on a cube topology.
In this model, each client is connected directly to three other clients - the ones with which it shares an edge along the cube. When such a client finds a new best chromosome (with respect to only its own population) it chooses a random one of its neighbours, and sends the chromosome to that neighbour. Each client does this every 25 generations. At the master level, there are just two groups; every 25 generations, the best chromosome within the first group (i.e. from all populations in the first cube) is sent to a randomly chosen client from the second cube. The same then happens vice versa. Finally, we tested both 8-population and 16population examples of each model. In the 8-population cases, the details of T1 and T2 (using 4 groups of 2) are straightforward. In the case of T3, this resulted in using just one cube, and hence did not involve migration at a higher level than the cube itself.

\section{EXPERIMENTS}

\section{A. Test Functions}

We compare the three models by using six well-known test functions, and use two versions of each - the standard version, and the 'shifted' version in which the global optimum is subject to random translation, rendering the function less decomposable. In Table I we indicate the functions used, and also indicate the target fitness. In most cases this is the global optimum. In all experiments, the functions had a dimension of 30 (i.e. 30 parameters).

TABLE I. FUNCTIONS USED IN EXPERIMENTS: TARGET FITNESSES

\begin{tabular}{|c|c|c|}
\hline Function & Shifted & Not Shifted \\
\hline Sphere & 0 & 0 \\
\hline Rosenbrock & 3.5 & 0 \\
\hline Schwefel & $5 \mathrm{E} 10^{-7}$ & 0 \\
\hline Rastrigin & 0 & 0 \\
\hline Griewangk & 0 & 0 \\
\hline Ackley & 0 & 0 \\
\hline
\end{tabular}

We tested each model (T1, T2 and T3) by running 20 independent trials on each of these 12 test functions, and doing this for each of an 8-population and 16-population case. We first indicate the raw results in terms of success rates - i.e. the number of times that each set of 20 runs resulted in finding a globally optimal chromosome. Note that there is no particular prior expectation that this will be large (or even above 0) in many cases, however the dynamics of parallel search, in conjunction with the operators chosen from [7], can be highly effective. 
TABLE II. SUCCESS RATES (OUT OF 20) FOR 8-POPULATION VERSIONS (1-CLIENT INDICATES SINGLE POPULATION SERIAL METHOD WITH EQUIVALENT OVERALL POPULATION SIZE): NON-SHIFTED AND SHIFTED FUNCTIONS

\begin{tabular}{|l|c|c|c|c||}
\hline Function & 1-client & T1 & T2 & T3 \\
\hline Sphere & $18(20)$ & $19(18)$ & $18(20)$ & $18(20)$ \\
\hline Rosenbrock & $10(14)$ & $18(20)$ & $18(20)$ & $18(18)$ \\
\hline Rastrigin & $20(12)$ & $20(18)$ & $20(17)$ & $20(18)$ \\
\hline Schwefel & $14(10)$ & $11(17)$ & $18(17)$ & $11(17)$ \\
\hline Griewangk & $20(14)$ & $18(18)$ & $20(20)$ & $18(17)$ \\
\hline Ackley & $20(16)$ & $20(19)$ & $20(20)$ & $18(18)$ \\
\hline
\end{tabular}

Table II shows the success rates for both non-shifted and shifted versions of the functions for the 8-population versions of each of the models tested in this paper. For example, on the Rastrigin function, model $\mathrm{T} 1$ achieved the target fitness on the non-shifted case in 11 runs out of 20 , and achieved target fitness in the shifted case in 17 runs out of 20. All models perform well in comparison with a standard single-population EA (which otherwise uses the same operators and overall population size). This is not surprising, although it is important to confirm. The performance advantage over the serial model (the "1-client" column) is more pronounced when we consider the shifted versions of the functions. In terms of success rates, the relative performances of $\mathrm{T} 1, \mathrm{~T} 2$ and $\mathrm{T} 3$ are very close, but perhaps $\mathrm{T} 2$ seems to have the advantage.

TABLE III. SUCCESS RATES (OUT OF 20) FOR 16-POPULATION VERSIONS (1-CLIENT INDICATES SINGLE POPULATION SERIAL METHOD WITH EQUIVALENT OVERALL POPULATION SIZE): NON-SHIFTED AND SHIFTED FUNCTIONS

\begin{tabular}{|l|c|c|c|c||}
\hline Function & 1-client & T1 & T2 & T3 \\
\hline Sphere & $18(20)$ & $20(20)$ & $20(20)$ & $20(20)$ \\
\hline Rosenbrock & $10(14)$ & $20(20)$ & $20(20)$ & $20(20)$ \\
\hline Rastrigin & $20(12)$ & $20(18)$ & $20(20)$ & $20(20)$ \\
\hline Schwefel & $14(10)$ & $16(17)$ & $17(18)$ & $15(18)$ \\
\hline Griewangk & $20(14)$ & $20(20)$ & $20(20)$ & $20(20)$ \\
\hline Ackley & $20(16)$ & $20(20)$ & $20(20)$ & $20(20)$ \\
\hline
\end{tabular}

Table III shows us the results for the 16-population versions of these models. Generally the performance of T1, $\mathrm{T} 2$ and $\mathrm{T} 3$ are all a little improved, with T2 perhaps maintaining a slight advantage, but with no statistical significance based on these data alone. The "1-client" column is repeated here for convenience, but reflects the same benchmark comparison experiment reported in table II.
In table IV we can see the mean execution times of the runs that were successful in reaching target fitness. Now we can see a much clearer advantage for $\mathrm{T} 2$, which seems to outperform $\mathrm{T} 1$ and $\mathrm{T} 3$ in each case.

TABLE IV. MEAN EXECUTION TIMES (MS) OF SUCCESSFUL RUNS (AVERAGED OVER ALL SUCCESSFUL RUNS) FOR 8-POPULATION VERSIONS (1-CLIENT INDICATES SINGLE POPULATION SERIAL METHOD WITH EQUIVALENT OVERALL POPULATION SIZE): NON-SHIFTED AND SHIFTED FUNCTIONS

\begin{tabular}{|l|c|c|c|c||}
\hline Function & 1-client & T1 & T2 & T3 \\
\hline Sphere & 72515 & 2261 & $\mathbf{1 5 6 5}$ & 1802 \\
& $(5.14 \mathrm{e} 6)$ & $(138503)$ & $\mathbf{( 1 1 2 7 1 2 )}$ & $(137969)$ \\
\hline & 375703 & 246484 & $\mathbf{1 9 3 9 8 3}$ & 313005 \\
Rosenbrock & $(1.01 \mathrm{e} 7)$ & $(1.76 \mathrm{e} 6)$ & $\mathbf{( 1 . 4 0 e 6 )}$ & $(1.80 \mathrm{e} 6)$ \\
\hline & 35197 & 2162 & $\mathbf{1 5 6 2}$ & 2172 \\
Rastrigin & $(1.44 \mathrm{e} 6)$ & $(153054)$ & $\mathbf{( 1 1 4 1 2 0 )}$ & $(132423)$ \\
\hline & $1.96 \mathrm{e} 6$ & 34059 & $\mathbf{2 5 3 1 6}$ & 49369 \\
Schwefel & $(1.59 \mathrm{e} 7)$ & $(528545)$ & $\mathbf{( 4 2 6 0 9 4 )}$ & $(713984)$ \\
\hline & 77949 & 2233 & $\mathbf{1 5 4 2}$ & 1856 \\
Griewangk & $(1.35 \mathrm{e} 6)$ & $(165531)$ & $\mathbf{( 1 4 3 7 9 3 )}$ & $(163831)$ \\
\hline & 75139 & 2031 & $\mathbf{2 0 0 0}$ & 2359 \\
Ackley & $(1.30 \mathrm{e} 6)$ & $(141634)$ & $\mathbf{( 1 3 2 8 1 2 )}$ & $(146466)$ \\
\hline
\end{tabular}

In tables V and VI, we can see the speedup fractions for all cases. These simply divide the mean execution time of successful runs in the serial case by the mean execution time in the parallel model case. The tables provide these data for each function, and the best speedup for each function is given in bold. The final line provides an indicative "mean speedup" over all functions.

TABLE V. SPEeduPS Of THE PARAllel ARChitectures COMPARED WITH SERIAL EA - 8-POPULATION MODELS

\begin{tabular}{|c|c|c|c||c|c|c|}
\hline \multirow{2}{*}{ Function } & \multicolumn{3}{|c||}{ Not Shifted } & \multicolumn{3}{c|}{ Shifted } \\
\cline { 2 - 7 } & $\mathrm{T} 1$ & $\mathrm{~T} 2$ & $\mathrm{~T} 3$ & $\mathrm{~T} 1$ & $\mathrm{~T} 2$ & $\mathrm{~T} 3$ \\
\hline Sphere & 32 & $\mathbf{4 6}$ & 40 & 37 & $\mathbf{4 6}$ & 37 \\
\hline Rosenbrock & $\mathbf{2}$ & $\mathbf{2}$ & 1 & 6 & $\mathbf{7}$ & 6 \\
\hline Rastrigin & $\mathbf{2 3}$ & $\mathbf{2 3}$ & 16 & 9 & $\mathbf{1 3}$ & 11 \\
\hline Schwefel & 58 & $\mathbf{7 8}$ & 40 & 19 & $\mathbf{2 3}$ & 14 \\
\hline Griewangk & 35 & $\mathbf{5 1}$ & 42 & 8 & $\mathbf{9}$ & 8 \\
\hline Ackley & 37 & $\mathbf{3 8}$ & 32 & 9 & $\mathbf{1 0}$ & 9 \\
\hline Mean & 30.65 & $\mathbf{4 6 . 7 6}$ & 32.55 & 14.72 & $\mathbf{1 7 . 9 9}$ & 14.13 \\
\hline
\end{tabular}


TABLE VI. SPEEDUPS OF THE PARALLEL ARCHITECTURES COMPARED WITH SERIAL EA - 16-POPULATION MODELS

\begin{tabular}{|c|c|c|c||c|c|c|}
\hline \multirow{2}{*}{ Function } & \multicolumn{3}{|c||}{ Not Shifted } & \multicolumn{3}{c|}{ Shifted } \\
\cline { 2 - 7 } & $\mathrm{T} 1$ & $\mathrm{~T} 2$ & $\mathrm{~T} 3$ & $\mathrm{~T} 1$ & $\mathrm{~T} 2$ & $\mathrm{~T} 3$ \\
\hline Sphere & $\mathbf{8 7 . 5 5}$ & $\mathbf{9 3 . 2 1}$ & $\mathbf{8 9 . 7 8}$ & 100.40 & $\mathbf{1 1 0 . 9 1}$ & 103.19 \\
\hline Rosenbrock & 9.38 & $\mathbf{1 0 . 0 7}$ & 7.28 & 13.48 & $\mathbf{1 8 . 3 6}$ & 14.09 \\
\hline Rastrigin & $\mathbf{4 8 . 2 3}$ & $\mathbf{5 1 . 2 0}$ & $\mathbf{4 2 . 1 8}$ & 25.63 & $\mathbf{3 2 . 7 2}$ & 25.89 \\
\hline Schwefel & 11.61 & $\mathbf{2 2 . 9 3}$ & 15.99 & 15.59 & $\mathbf{1 6 . 4 0}$ & 11.30 \\
\hline Griewangk & 90.70 & $\mathbf{1 1 1 . 0 4}$ & 103.09 & 33.01 & $\mathbf{4 5 . 3 0}$ & 23.06 \\
\hline Ackley & 92.86 & $\mathbf{1 1 1 . 0 5}$ & 93.55 & 28.64 & $\mathbf{4 1 . 9 7}$ & 25.03 \\
\hline Mean & 57.23 & $\mathbf{6 6 . 5 8}$ & $\mathbf{5 8 . 6 4}$ & 36.13 & $\mathbf{4 4 . 2 8}$ & 33.76 \\
\hline
\end{tabular}

It is clear that model T2 is the most successful in terms of speed of finding global optima in each case.

\section{CONCLUding Discussion}

We have argued that distributed evolutionary algorithms (dEAs) are of ever-increasing interest and importance for a variety of reasons. It is well known that parallelized optimization can provide more advantages than simply speed of execution; meanwhile the design of a distributed, asynchronous architecture leads to opportunities for managing exploration and exploitation in ways that simply cannot be done in serial, and these can lead to better results for the same overall number of function evaluations. Given that parallel hardware resources are becoming more common and everyday, it is therefore clear that we need to understand how to design dEAs to optimal effect.

So far, however, there has been relatively little in terms of exploration of the vast number of potential architectures and migration strategies (for example) in the broad space of possible dEAs. In this paper we have compared some simple dEA topologies and interaction schemes. One was a straightforward case of dividing the population into $N$ subpopulations, where a master process distributed new best chromosomes to the current worst subpopulation whenever a new best was found. The second was a slight variation on the first, in which the individual populations were each groups of two subpopulations, which communicated their best chromosomes regularly to each other, with the "best to worst" strategy operating at the level above these pairs of populations. The third model was based on Albe et al's cube topology [7] and also used the a similar migration scheme.

Interestingly, we found that the simple variation between model 1 and model 2 led to a significant difference in performance, which was clearly seen in the average speedups, in both the 8-population and 16-population cases. Model 2 appeared more successful than Model-3, inspired by Alba et al's GD-RGCA, on the functions tested.

One possible conclusion is that GD-RCGA (and perhaps other current models, might be enhanced by adopting aspects of the interaction strategy and topology used in model 2. This is one idea that we expect to examine in future work. Also in future work we will more systematically explore the design and parameter settings of model 2 , to determine what seems to lead to its outperformance of model 1 . We expect it will be interesting to explore this, for example, by tracking the generation and behaviour of niches in the fitness landscape as they emerge and are shared between processors.

\section{REFERENCES}

[1] T. Maruyama and T. Hirose and A. Konagaya, A Fine-Grained Parallel Genetic Algorithm for Distributed Parallel Systems. In proceedings of the 5th International Conference on Genetic Algorithms, pp. 184-190, 1993.

[2] S. Baluja, Structure and Performance of Fine-Grain Parallelism in Genetic Search. In proceedings of the 5th International Conference on Genetic Algorithms, pp. 155-162, 1993.

[3] H. M"uhlenbein, Parallel genetic algorithms, population genetics and combinatorial optimization. In proceedings of the 3th International Conference on Genetic Algorithms, pp. 416-421, 1989.

[4] B. Manderick and P. Spiessens, Fine-grained parallel genetic algorithms. In proceedings of the 3th International Conference on Genetic Algorithms, pp. 428-433, 1989.

[5] T. Starkweather and L. Darrell Whitley and Keith E. Mathias, Optimization Using Distributed Genetic Algorithms. In proceedings of the 1st Workshop on Parallel Problem Solving from Nature, pp. 176-185, 1991.

[6] V. S. Gordon and L. D. Whitley, Serial and Parallel Genetic Algorithms as Function Optimizers. In proceedings of the 5th International Conference on Genetic Algorithms, pp. 177-183, 1993.

[7] E. Alba, F. Luna, A.J. Nebro, A.J., J.M. Troya Parallel heterogeneous genetic algorithms for continuous optimization. Parallel Computing. Vol 30, No 5-6, pp. 699-719. May/June 2004

[8] L. D. Whitley, "The GENITOR algorithm and selection pressure: Why rank-based allocation of reproductive trials is best," in Proc. $3 r d$ Int. Conf. on Genetic Algorithms. San Mateo, CA: Morgan Kaufmann, 1989, pp. 116-121.

[9] Uwe Aickelin, Larry Bull: Partnering Strategies For Fitness Evaluation In A Pyramidal Evolutionary Algorithm. GECCO 2002: 263-270.

[10] Muhlenbein, H. and Schlierkamp-Voosen, D. (1993). Predictive models for the breeder genetic algorithm i. continuous parameter optimization. Evolutionary Computation, 1(1):25\{49. 